\title{
Perforation of a Gastric Tear during Esophageal Endoscopic Submucosal Dissection under General Anesthesia
}

\author{
Tomoaki Yamasaki, Yuhei Sakata, Takehisa Suekane and Hiroko Nebiki \\ Department of Gastroenterology, Osaka City General Hospital, Osaka, Japan
}

Mallory-Weiss tears (MWT) are occasionally encountered during endoscopic procedures. Esophageal endoscopic submucosal dissection (ESD) is widely performed under general anesthesia to avoid unexpected body movements. We present the case of a 68-year-old woman with squamous cell carcinoma. Although ESD was performed under general anesthesia, a gastric perforation at the MWT caused by gastric inflation was observed after the procedure. The perforation was closed endoscopically, and she was discharged without any sequelae. Although general anesthesia is useful for esophageal ESD, it should be noted that it can cause MWT, and in rare cases, gastric perforation, due to gastric inflation during the procedure. Clin Endosc 2021;54:916-919

Key Words: Endoscopic submucosal dissection; Gastric perforation; General anesthesia; Mallory-Weiss tear

\section{INTRODUCTION}

Esophageal endoscopic submucosal dissection (ESD) is widely performed for early stage neoplasms of the esophagus. ${ }^{1}$ With various innovations such as submucosal injection ${ }^{2}$ and traction methods, ${ }^{3}$ ESD can now be performed safely. Patients with esophageal carcinoma usually have a history of excessive alcohol consumption. Thus, sedation with benzodiazepines can occasionally cause disinhibition during an ESD, which may render the procedure more difficult and unsafe. For this reason, general anesthesia has recently been used for esophageal ESD. ESD under general anesthesia is suitable and safe, ${ }^{4}$ however, some peculiar complications such as a sore throat due to intubation, malignant hyperthermia, and hypothermia occur during ESD under anesthesia. Herein, we report a case

Received: August 14, 2020 Revised: September 15, 2020

Accepted: September 15, 2020

Correspondence: Tomoaki Yamasaki

Department of Gastroenterology, Osaka City General Hospital, 2-13-22, Miyakojima-hondori, Miyakojima-ku, Osaka 534-0021, Japan

Tel: +81-6-6929-1221, Fax: +81-6-6929-1090, E-mail: tomorrow_aaa@yahoo. co.jp

ORCID: https://orcid.org/0000-0003-0251-6927

(cc) This is an Open Access article distributed under the terms of the Creative Commons Attribution Non-Commercial License (http://creativecommons.org/ licenses/by-nc/3.0) which permits unrestricted non-commercial use, distribution, and reproduction in any medium, provided the original work is properly cited. of perforation of a gastric tear during an esophageal ESD under general anesthesia.

\section{CASE REPORT}

A 68-year-old woman with esophageal squamous cell carcinoma was referred to our institution after the tumor was detected during a routine esophagogastroduodenoscopy (EGD). Although she did not take any medications, she had a history of excessive alcohol consumption. On magnifying endoscopy with narrow band imaging and endoscopic ultrasonography, the lesion was located within the mucosal layer in the upper esophagus. Thus, for removal, ESD was scheduled and was started under general anesthesia with desflurane, propofol, and remifentanil hydrochloride. Rocuronium bromide was also administered as a muscle relaxant.

ESD was performed conventionally using carbon dioxide insufflation. Preoperative EGD showed no gastric atrophy in this case.

When an endoscope was advanced into the stomach for suction of the liquid and the air during the dissection, gastric tears were observed without any evidence of perforation or bleeding. Complete resection was achieved after $44 \mathrm{~min}$, and the size of the resected specimen was $25 \times 24 \mathrm{~mm}$, and the size of the lesion was $13 \times 8 \mathrm{~mm}$. After retrieving the specimen, 


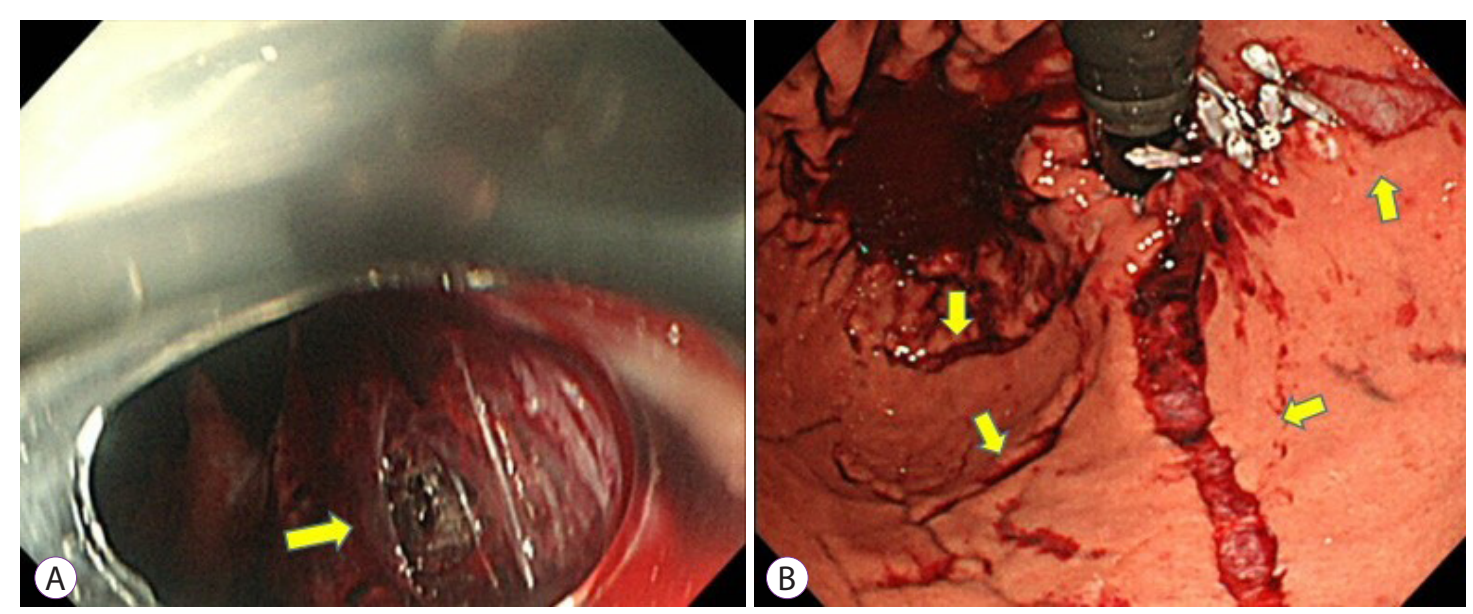

Fig. 1. (A) Perforation in the tear at the gastric upper body (arrow). (B) Multiple gastric lacerations in the stomach and closure of the perforation site using endoclips.

the endoscope was reinserted into the stomach to confirm the tears. Detailed observation revealed multiple gastric tears, one of which was bleeding, with a perforation in the lesser curvature of the gastric upper body and fornix. The perforation site was immediately closed endoscopically using endoclips (Fig. 1). Computed tomography was performed to evaluate the perforation, which revealed free air in the peritoneum and retroperitoneum. Free air was considered to be caused by the gastric perforation because there was no perforation or injury to the muscle layer in the ESD site (Fig. 2), and pneumomediastinum and subcutaneous emphysema were not observed (Fig. 3).

The patient was treated conservatively with intravenous antibiotics (1 $\mathrm{g}$ cefazolin, twice daily for 7 days). Moreover,

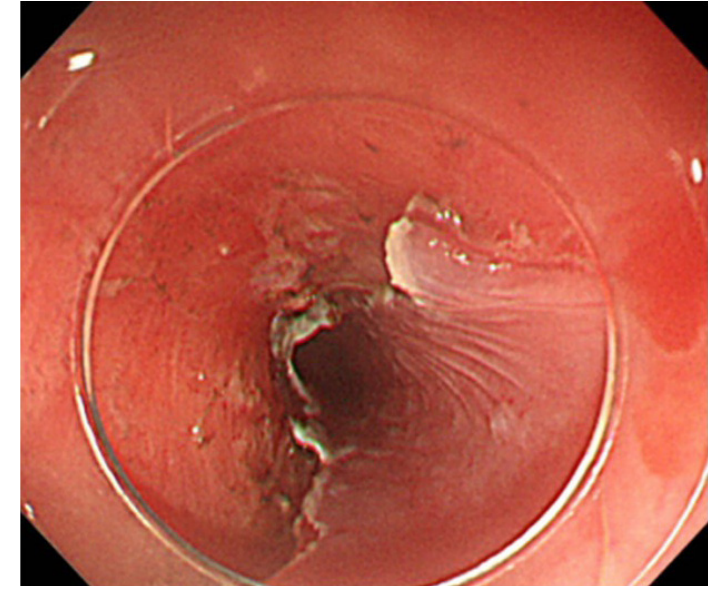

Fig. 2. No perforation or injury to the muscle layer was observed at the ulcer bed after endoscopic submucosal dissection.
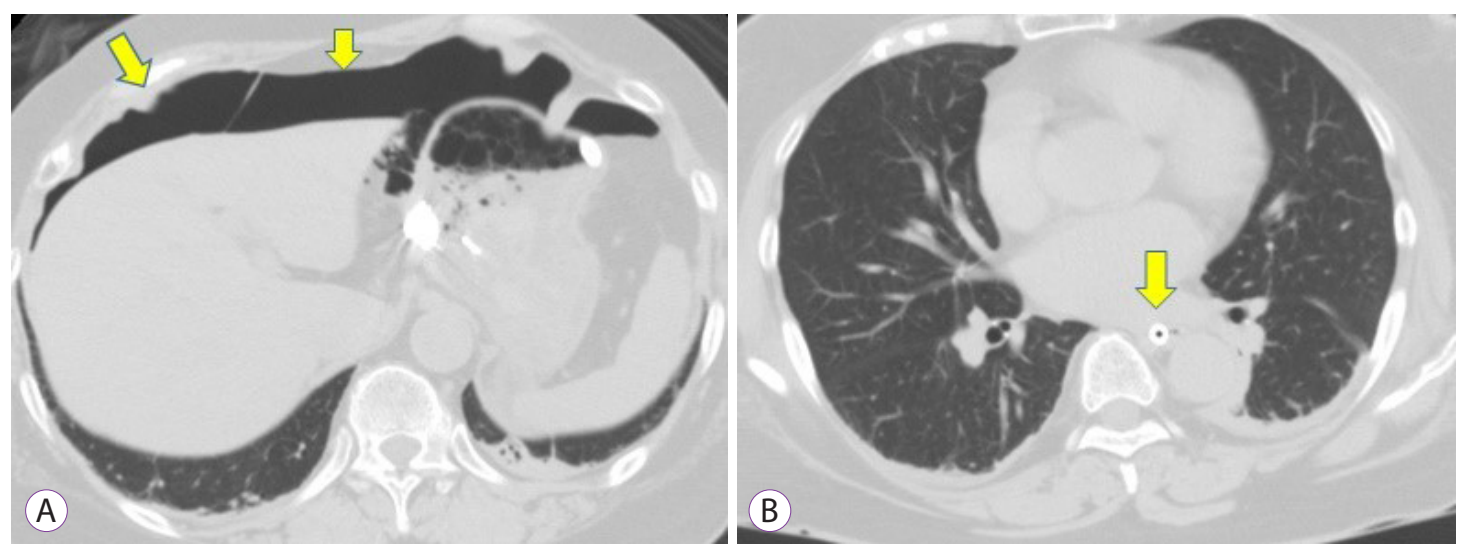

Fig. 3. Post-procedural computed tomography. (A) Peritoneal gas was observed (arrow). (B) No pneumomediastinum was observed (a nasogastric tube was inserted, arrow). 


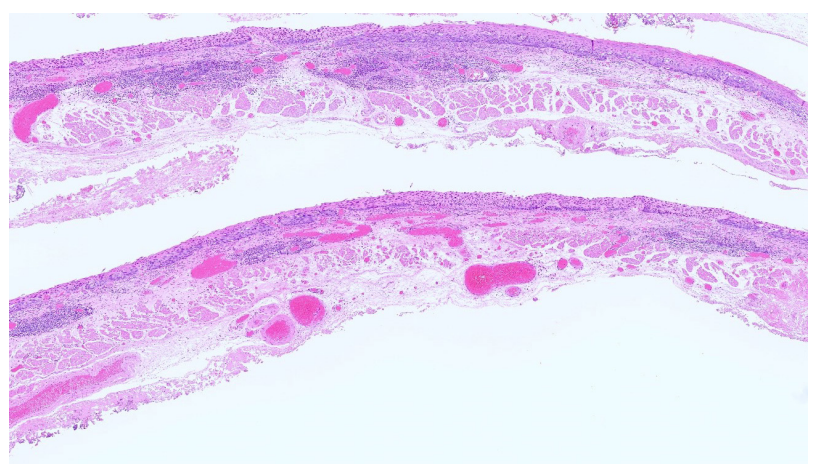

Fig. 4. Microscopic image of resected specimen. There was no evidence of resected muscle layer (hematoxylin and eosin stain $\times 10$ ).

she started drinking water and began a diet on the 5th and 6th days post-ESD, respectively. The histology of the resected lesion revealed a squamous cell carcinoma limited to the intraepithelial layer with no evidence of the resected muscle layer (Fig. 4). The patient was discharged on the 8th postoperative day without any sequelae.

\section{DISCUSSION}

Mallory-Weiss tears (MWT) occur rarely as a complication of ESD, with an incidence of $0.07-0.49 \% .{ }^{5}$ Iatrogenic tears usually occur in the atrophic stomach, mostly in the right lateral wall of the esophagus and the lesser curvature of the gastric upper body. ${ }^{6}$ In our case, multiple lacerations also occurred at the lesser curvature of the gastric upper body and fornix. Chen et al. ${ }^{7}$ reported 20 cases (5.4\%) of MWT detected during an esophageal ESD under general anesthesia, with only one case of MWT perforation. In addition, female sex and procedure time longer than $88.5 \mathrm{~min}$ were shown to be independent risk factors (odds ratio $=5.270$ and 3.953, respectively) for MWT during esophageal ESD. Although the sex of our patient was female, the procedure time was not long.

Since esophageal ESD requires a precise technique and has a high complication rate, reliable sedation is needed to perform an esophageal ESD. Conscious sedation is suitable for endoscopic procedures. However, since alcohol consumption which causes inadequate sedation, including disinhibition, is the most important risk factor for esophageal squamous cell carcinoma, conventional sedation may be insufficient for esophageal ESD. ${ }^{8}$ The usefulness of general anesthesia has been reported for esophageal ESD., ${ }^{4,9}$ General anesthesia has peculiar complications, including our case.

Esophageal ESD tends to cause gastric inflation because gas suction in the esophagus does not effectively reduce the gas in the stomach. This necessitates repeated suction of the gas in the stomach during an esophageal ESD. Gastric inflation under general anesthesia will not only cause discomfort to the patients but will also lessen the diaphragmatic movement and reduce the tidal volume of ventilation. General anesthesia may also induce gastric inflation due to diminished tonus of the gastric muscles. Additionally, less belching under general anesthesia can also cause an increase in the volume of the gastric gas.

Carbon dioxide, due to its rapid absorption, is useful to avoid gas-related complications, compared to air. ${ }^{10}$ Although the severity of complications such as perforation and pneumomediastinum are diminished by the use of carbon dioxide, it does not contribute to the prevention of MWT caused by gastric inflation. This is because its absorption in the stomach and intestine is rapid but not instantaneous. Therefore, both monitoring of the volume of the gastric gas and a frequent gas suction are important to reduce gas-induced complications during esophageal ESD.

In conclusion, although general anesthesia is useful for esophageal ESD, MWT induced by gastric overinflation under general anesthesia is a complication that cannot be ignored. The frequent suction of gastric gas during the procedure may diminish this type of complication.

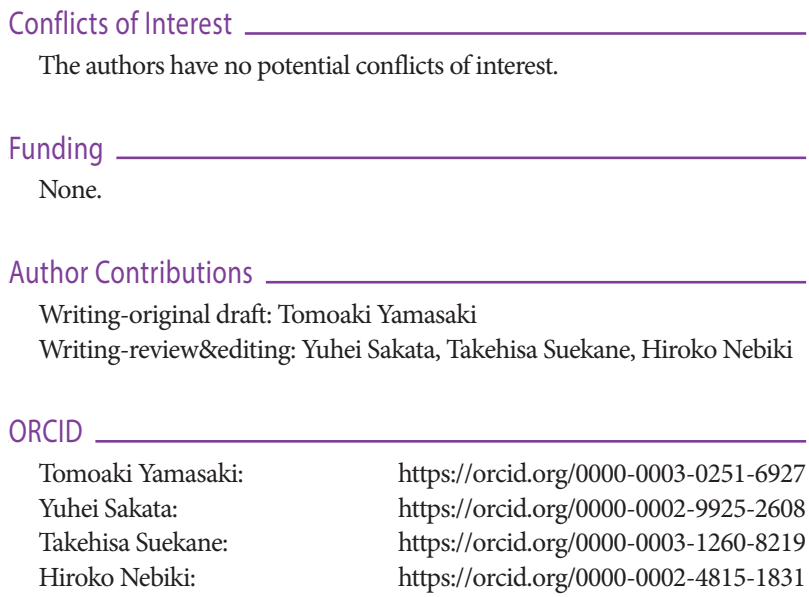

https://orcid.org/0000-0003-0251-6927 https://orcid.org/0000-0002-9925-2608 https://orcid.org/0000-0003-1260-8219 https://orcid.org/0000-0002-4815-1831

\section{REFERENCES}

1. Fujishiro M, Yahagi N, Kakushima N, et al. Endoscopic submucosal dissection of esophageal squamous cell neoplasms. Clin Gastroenterol Hepatol 2006;4:688-694.

2. Yandrapu H, Desai M, Siddique S, et al. Normal saline solution versus other viscous solutions for submucosal injection during endoscopic mucosal resection: a systematic review and meta-analysis. Gastrointest Endosc 2017;85:693-699. 
3. Yoshida M, Takizawa K, Nonaka S, et al. Conventional versus traction-assisted endoscopic submucosal dissection for large esophageal cancers: a multicenter, randomized controlled trial (with video). Gastrointest Endosc 2020;91:55-65.e2.

4. Hamada K, Kawano K, Yamauchi A, et al. Efficacy of endoscopic submucosal dissection of esophageal neoplasms under general anesthesia. Clin Endosc 2019;52:252-257.

5. Penston JG, Boyd EJ, Wormsley KG. Mallory-Weiss tears occurring during endoscopy: a report of seven cases. Endoscopy 1992;24:262-265.

6. Okada M, Ishimura N, Shimura S, et al. Circumferential distribution and location of Mallory-Weiss tears: recent trends. Endosc Int Open 2015;3:E418-E424.

7. Chen W, Zhu XN, Wang J, Zhu LL, Gan T, Yang JL. Risk factors for Mal-
lory-Weiss tear during endoscopic submucosal dissection of superficial esophageal neoplasms. World J Gastroenterol 2019;25:5174-5184.

8. Ominami M, Nagami Y, Shiba M, et al. Comparison of propofol with midazolam in endoscopic submucosal dissection for esophageal squamous cell carcinoma: a randomized controlled trial. J Gastroenterol 2018;53:397-406.

9. Yamashita K, Shiwaku H, Ohmiya T, et al. Efficacy and safety of endoscopic submucosal dissection under general anesthesia. World J Gastrointest Endosc 2016;8:466-471.

10. Takada J, Araki H, Onogi F, et al. Safety and efficacy of carbon dioxide insufflation during gastric endoscopic submucosal dissection. World J Gastroenterol 2015;21:8195-8202. 